\title{
Estimation Research on the RCS of a Complex \& Electrically Large Size Target
}

\author{
Shengliang $\mathrm{Hu}$ \\ College of Electronic Engineering \\ Naval University of Engineering \\ Wuhan, China \\ hgdhsl@sina.com \\ Zhong Liu \\ College of Electronic Engineering \\ Naval University of Engineering \\ Wuhan, China
}

\author{
Tao Liu \\ College of Electronic Engineering \\ Naval University of Engineering \\ Wuhan, China \\ liutao1018@sina.com
}

\begin{abstract}
Radar scattering characteristics is an important researching content of military target. It is always very difficult to deal with simulation of electromagnetism field, especially to analyze the monostatic RCS characteristics. Aiming at a kind of complex \& electrically large size target, this paper reasonably estimated its multi-point monostatic RCS using smaller resources by adopting the electromagnetic calculation software FEKO. This work succeeded on the analysis and optimization choice to typical high frequency algorithm theory, combined with the prediction precision and efficiency. This project has an important value in engineering application.
\end{abstract}

Keywords-RCS, Estimation, Electrically Large Size, geometrical optics(GO), physical optics(PO), algorithmg

\section{INTRODUCTION}

Radar scattering characteristics is an important researching content of military target. It is important to estimate the target RCS quickly and accurately to engineering application. In recent decades, with the technology of radar and computer rapid developed, the estimate method and technology of the RCS also made breakthrough progress. Successively developed the Method of Moments (MOM), the Multilevel Fast Multipole Method (MLFMM), Finite Difference Time Domain(FDTD), physical optics(PO) method, geometrical optics(GO)method and other typical algorithm, released excellent electromagnetic calculation software, such as XPATCH, GRECO and FEKO. $^{[1][2][3]}$

Numerous texts have detail researched on the RCS estimation method to specific structural target, and evaluated the effectiveness through self-designing program. But these studies are more time-consuming and exhausting generally, have no universal applicability in engineering application.

FEKO and other excellent electromagnetic calculation software, improved the RCS estimating efficiency greatly to electrically large size target. They have the universal application to all kinds of target based on the current mainstream algorithm optimized, achieve the calculation \& estimation of target's RCS through the following steps, such as establishing model, setting parameters, selecting algorithm, calculating and displaying results. But also having these problems such as computing resource requirement is too high, estimation accuracy declining, if the algorithm choosing is unreasonable.

Aiming at a kind of complex \& electrically large size target, this paper reasonably estimated its multi-point monostatic RCS using smaller resources by FEKO, based on the analysis and optimization choice to typical high frequency algorithm theory, combined with the prediction precision and efficiency .

\section{ENGINEERING DESCRIPTION AND ALGORITHM ANALYSIS}

\section{A. Engineering Description}

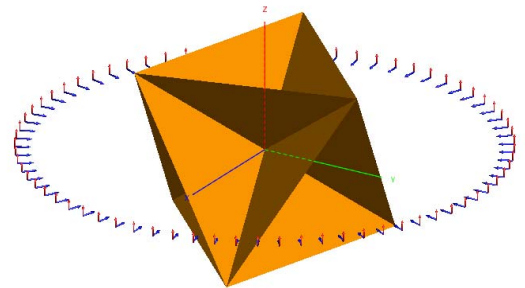

Figure 1. a Complex Electrically Large Size Structure

A complex Electrically Large size target as shown in figure 1 , it is composed of eight standard triangle reflectors with ideal conductor. Triangle Angle reflector vertical side $a=2500 \mathrm{~cm}$.Estimating its monostatic RCS excited by plane wave $\left(\lambda=2 \mathrm{~cm}, \varphi=\left[0^{\circ}, 360^{\circ}\right], \theta=\left[30^{\circ}, 90^{\circ}\right]\right)$.

\section{B. Algorithm Analysis}

This project is a typical RCS prediction problem of large electrically size target. It is always very difficult to deal with simulation of electromagnetism field, especially to analyze the monostatic RCS characteristics. Corresponding to multiple scanning angle, the calculation is a very huge amount work. So, for this kind of problem, calculation software should meet the following requirements: (i) Basing on the integral equation. (ii) Containing integral equation method, such as MLFMM, $\mathrm{ACA}$, etc. (iii) Adopting high-frequency algorithm, such as GO, PO etc. 
FEKO is a electromagnetic field simulation software, can be used for various types of electromagnetic field analyses involving objects of arbitrary shapes. Based on the classical MOM and the integration of MLFMM, PO, GO, UTD,FEM and its hybrid algorithm, achieves the matrix \& PO,\&GO, \& UTD union, has an important goal of estimate RCS application value for great electrically size, suitable for this paper engineering requirements. The method of PO is not coalesced with GO in the software, so it is a premise to estimate the RCS efficiently and accurately by choosing the approximate highfrequency algorithm reasonably. ${ }^{[5] \sim[7]}$

\section{ALGORITHM OPTIMIZATION}

Because the target is a many reflective planar structure, GO algorithm is suitable theoretically. Here making applicability comparison and validating analysis between PO and GO.

\section{A. The Benchmark Algorithm Selection}

Here selecting classic triangle reflector structures as the research object, shown in figure 2. The right side equal $23 \mathrm{cmand}$ the excitation incident( frequency equal $9.3 \mathrm{GHz}$ ) coming from horizontal direction( vertical polarization).MOM and MLFMM algorithm were used to calculate its monostatic RCS. The calculation results shown in figure 3 .We can see the curves drawn from the two algorithms match perfectly, and is consistent with the actual measurement value trend provided by the literature [8]. It shows the two algorithms all have accuracy result. The resource consumption of MLFMM method is much less of MOM to electrically large target's RCS prediction, so this paper adopt MLFMM as the benchmark algorithm.

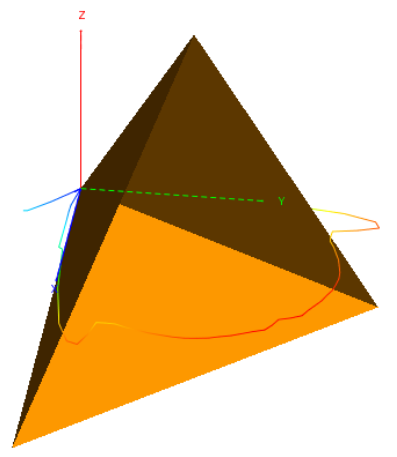

Figure 2. benchmark structures sketch map

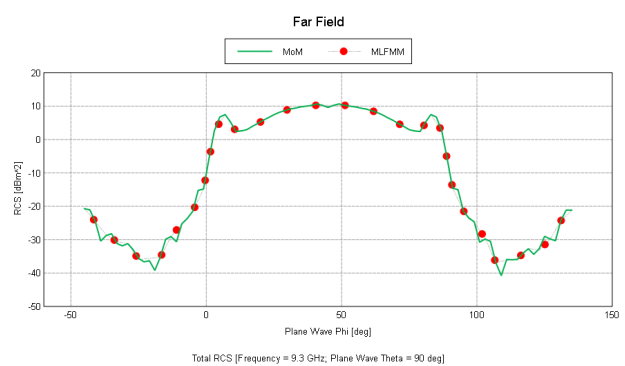

Figure 3. the monostatic RCS curve based on MLFMM \& MOM

\section{B. Model Simplified}

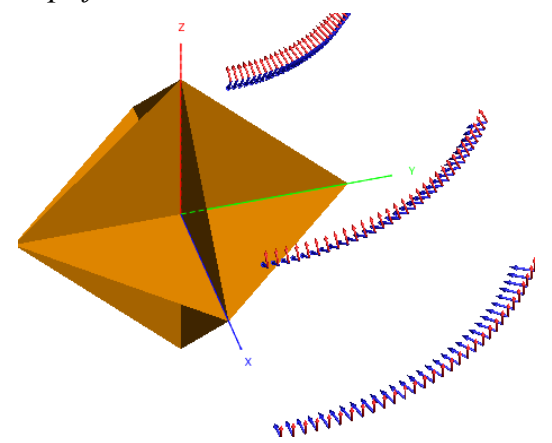

Figure 4. simplified model schemes

For the convenient research, set the right side equal $5 * \lambda(\lambda=2 \mathrm{~cm})$, calculating the monostatic RCS. The result shown in figure 4.

$$
\text { Here, } \theta=\left[30^{\circ}, 90^{\circ}\right], \varphi=\left[0^{\circ}, 60^{\circ}\right] \text {. }
$$

\section{Estimated Accuracy Comparion}

The estimated results drawn from PO and GO are compared with MLFMM respectively.

1) estimate accuracy compared between PO \& MLFMM

The prediction graph of simplified model's RCS based on PO (single reflection) method and MLFMM method respectively shown in Figure 5.

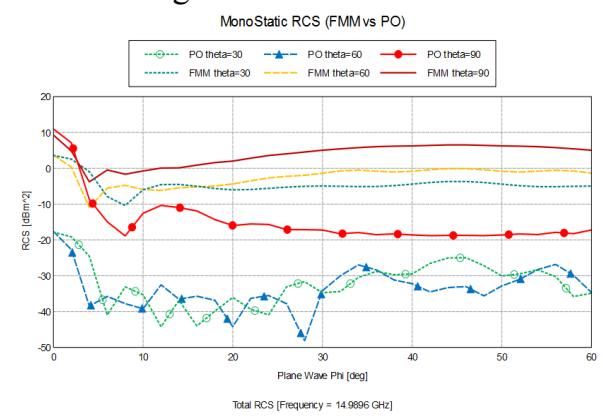

Figure 5. the monostatic RCS curve based on MLFMM \& PO (single reflection)

The dotted line is calculation results of MLFMM, solid is of PO. We can see the estimated results of PO has larger error with the MLFMM. To improve the accuracy, we can consider installing multiple reflections theoretically. Here, using 2-times reflection.

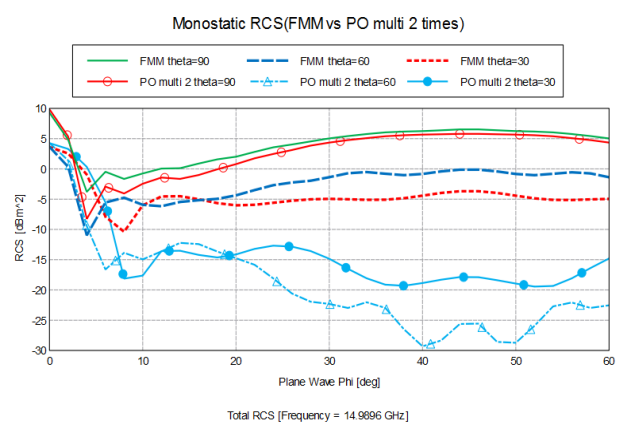

Figure 6. the monostatic RCS curve based on MLFMM \& PO (2-times reflection) 
Figure 6 shows the accuracy is improved greatly comparing with single reflection, especially in horizontal plane.

2) estimated accuracy compared between PO\& MLFMM

The prediction graph of simplified model's RCS based on GO method and MLFMM method respectively shown in Figure 7.

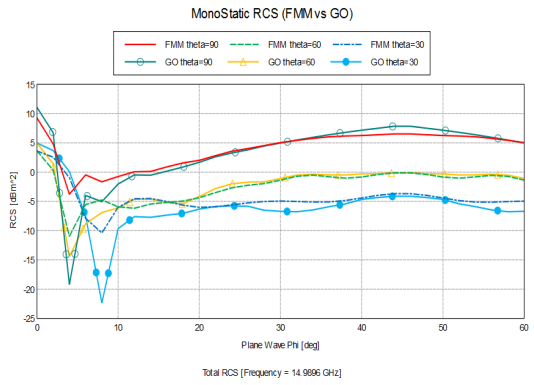

Figure 7. the monostatic RCS curve based on MLFMM \& GO

Dotted line is also of MLFMM, the solid line is of GO. We can see the curves drawn from the two algorithm match perfectly, so the prediction result of GO is reliable.

3) mesh dissection and $G O$ algorithm

In order to analyze the influence of GO algorithm caused by different mesh dissection, the two mesh dissection of $\lambda / 4$ and $\lambda / 2$ were adopt respectively. The estimated RCS curves shown in figure 8 and the resource requirements statistics list in table 1 based on the two mesh dissection.

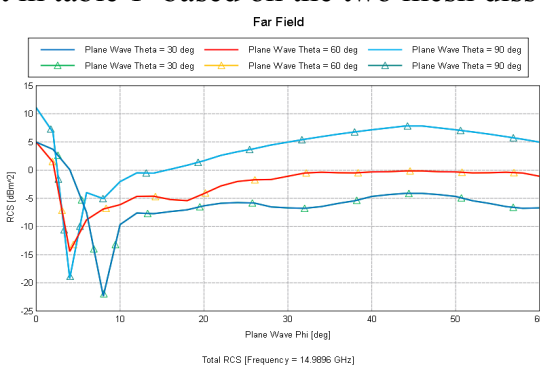

Figure 8. the monostatic RCS curve based on different mesh dissection

TABLE I. THE RESOURCE REQUIREMENTS STATISTICS

\begin{tabular}{|c|c|c|c|}
\hline Grid length & Grid number & memory & time \\
\hline$\lambda / 4$ & 6620 & $4.1 \mathrm{M}$ & $955 \mathrm{~S}$ \\
\hline$\lambda / 2$ & 1648 & $1.943 \mathrm{M}$ & $257 \mathrm{~S}$ \\
\hline
\end{tabular}

Figure 8 shows that for such planar structures, GO algorithm is insensitive to the mesh dissection density. Table 1 shows that with grid number increasing, calculation resources consumed more greater. So, for high frequency RSC computation, we can try shrinkage-reducing grid precision scale, and the result still credible.

\section{Consumes resources comparing}

TABLE II. RESOURCE CONSUMPTION STATISTICS ON DIFFERENT ALGORITHMS

\begin{tabular}{|c|c|c|}
\hline Algorithm & Memory & Time \\
\hline MLFMM & $607 \mathrm{M}$ & $1.148 \mathrm{~h}$ \\
\hline
\end{tabular}

\begin{tabular}{|c|c|c|}
\hline PO(single reflection) & $10.7 \mathrm{M}$ & $32.9 \mathrm{~S}$ \\
\hline GO & $1.943 \mathrm{M}$ & $257 \mathrm{~S}$ \\
\hline PO (2-times reflection) & $81.35 \mathrm{M}$ & $5.176 \mathrm{~h}$ \\
\hline
\end{tabular}

Therefore, whether from the prediction accuracy or computing resource consumption, GO algorithm both has obvious advantages than $\mathrm{PO}$ for this paper.

\section{RCS ESTIMATION ON GO}

The monostatic RCS curve based on GO to 2.1 project shown in Figure9, and the resource consumption statistics listed in the table 3.

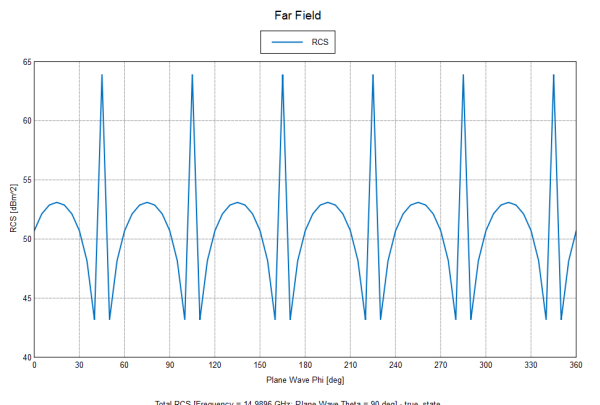

(a)

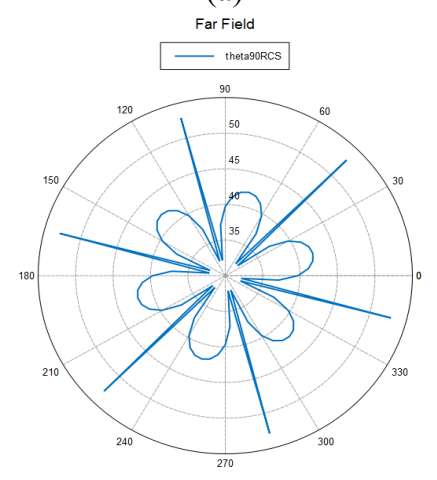

(b)

Figure 9. the monostatic RCS curve in horizontal plane $\left(\theta=90^{\circ}\right)$

TABLE III. RESOURCE CONSUMPTION STATISTICS

\begin{tabular}{|c|c|c|}
\hline Memory & Single-Point Time & Total Time \\
\hline $702.2 \mathrm{M}$ & $1.65 \mathrm{~h}$ & $120.8 \mathrm{~h}$ \\
\hline
\end{tabular}

From the results, we can know that the scattering characteristics present very obvious cyclical changes in horizontal plane. the trend and the actual geometric structure identical. Judging from the statistical results, the main resources are consumed in the ray tracing mainly, it can be accept in engineering.

\section{CONCLUSION}

Aiming at a kind of complex \& electrically large size target, this paper reasonably estimated its multi-point monostatic RCS using smaller resources by adopting the electromagnetic calculation software FEKO. This work succeeded on the analysis and optimization choice to typical high frequency algorithm theory, combined with the prediction 
precision and efficiency .This project has an important value in engineering application.

\section{REFERENCES}

[1] Nie Zaiping,Fang Dagang. Model-Theroy-Method\& Realization to the elector magnetism scatter of target \& environment(basement). National Defense Industry Press, 2009 (In Chinese).

[2] Zhuang Daowen, Yuan Naichang,Mo Jingjun and Liu Shaobing, RCS Estimate and Measure to Military Target. Science Press,2007 (In Chinese)

[3] HUANG Ji-jun, SUYi, YUWen-xian, Calculation of the Radar Cross Section of Targets Based on Triangular Object Model, Journal Of National University Of Defense technology,Vol.26 No.6 2004

[4] WANG Jie, HONG Wei.Fast calculation of wide angle RCS of 3-D objects, CHINESE JOURNAL OF RADIO SCIENCE, Vol.16, No.2June, 2001, 241-243.

[5] Yan Zhaowen,Su Donglin and Yuan Xiaomei. Example \& technology analysis of electormagnetisc field. National Water Press, 2009 (In Chinese).

[6] EM Software \& Systems-S.A. (Pty) Ltd. Getting Started Manual Suite 5.0.http://www.feko.info,July.2005.

[7] Chen Dexi,Yan Li,Wang Haiying. Emulational application by the Software FEKO in the Radar Cross Section. Ship Electronic Engineering. pp.25-128,ol.171,No.9,2008 (In Chinese).

[8] Zhao Jie, Yu Qun.Measure of The RCS .Modem Radar[J].1993.06:1-7 